\title{
Two Distinct c-ski cDNAs of Fish, Tilapia (Oreochromis aurea)
}

\author{
CHIU-JU HUANG, JER-YOUNG LIN, AND HUAI-JEN TSAI* \\ Institute of Fisheries Science, National Taiwan University, Taipei, Taiwan
}

\begin{abstract}
Two classes of tilapia c-ski cDNA (accession nos. AJ012011, AJ012012), designated as tskil and tski2, respectively encoded a 687 and a 714 AA protein and shared a $57 \%$ AA identity. Comparison with the Ski proteins of chickens, humans and Xenopus, tilapia TSki polypeptides shared a 60,57 , and $57 \%$ (TSki1) and 67, 63, and 61\% (TSki2) AA identity, respectively. The most and the least abundant c-ski mRNAs are located in the brain and the skeletal muscle, respectively. Both tski1 and tski2 were widely expressed in the adult tissues examined, but tski2 transcripts were at higher levels except in the ovary and oocytes: tskil transcripts were predominant in the ovary, whereas tski2 transcripts were predominant in the testes. In the oocytes, the tskil mRNA was a maternally-inherited stockpile that subsequently was degraded, so that the expression ratio of tskil to tski2 transcripts declined gradually as the fish developed from oocyte to 4-cm fry. Mol. Reprod. Dev. 54:223231. ๑ 1999 Wiley-Liss, Inc.
\end{abstract}

Key Words: cDNA; c-ski; fish; oncogene; Ski protein

\section{INTRODUCTION}

c-ski is proto-oncogene which encodes an $84 \mathrm{kDa}$ nuclear protein (Nagase et al., 1990). The c-Ski protein stimulates proliferation, induces morphologic transformation, and promotes myogenic differentiation on cultured quail embryo fibroblasts (Stavnezer et al., 1981; Li et al., 1986; Stavnezer et al., 1986; Colmenares and Stavnezer, 1989; Colmenares et al., 1991). Transgenic mice show a distinctive muscular phenotype due to a hypertrophic effect on the formation of the fast muscle IIb fibers and concomitant decrease in body fat (Sutrave et al., 1990). c-Ski activates a muscle-specific enhancer resulting in the transcriptional regulation of muscle differentiation (Engert et al., 1995). c-ski is widely expressed in many tissues, although some studies that investigated skeletal muscle in Xenopus and chicken found no evidence of c-ski expression (Sutrave et al., 1990; Sleeman and Laskey, 1993). Namciu et al. (1994) reported c-ski expression at a high level in the skeletal muscle of mouse embryos at 12.5 days of gestation. Knockout mice die at birth and show a defective closure of the rostral neural tube and a decrease in skeletal muscle (Berk et al., 1997). Recently, Nicol and Stavnezer (1998) demonstrated that c-Ski acts as a repressor rather than an activator, and depends on the cellular environment that allows c-Ski to bind to the GTCTAGAC site. Ichikawa et al. (1997) also reported that c-Ski functions as suppressor of the myogenic gene in proliferating myoblasts and as a potentiator in post mitotic myotubes. The fact that c-Ski apparently plays many important roles suggests that its functionality as a transcriptional factor may be modified by interactions with various other transcriptional factors (Tarapore et al., 1997). It would therefore appear to be impossible to understand clearly and completely the biological properties of c-Ski without studying c-Ski in more diverse species. However, only the cDNA-derived c-ski gene of chicken (Sutrave and Hughes, 1989), human (Nomura et al., 1989), mouse (Namciu et al., 1995), Xenopus (Sleeman and Laskey, 1993) and axolotl (Ludolph et al., 1995) have been defined. To date, no such definition has been available for the cellular c-ski gene of the lowest vertebrates, fish.

As a step toward providing this information, we herein describe two classes of c-ski cDNAs of tilapia (Oreochromis aurea), one of the most popularly farmed fish species around the world. These date provide a wider base of knowledge on the primary structure and the gene expression of fish c-ski.

\section{MATERIALS AND METHODS RNA Isolation and RT-PCR (Reverse Transcription PCR)}

RNAs were extracted from the whole bodies of tilapia fry $(1.6 \mathrm{~cm})$ using TRI reagent (Molecular Research Center). Polyadenylated RNA $(0.2 \mu \mathrm{g})$ isolated with a poly(A) mRNA Isolation System (Promega) was reversetranscribed using an oligo $(\mathrm{dT})_{12-18}$ primer and SuperScript reverse transcriptase (BRL). The single strand cDNA products were subjected to PCR with primers $1^{\prime} \mathrm{F}$ (5'ATCTSTGCTTYGTGGTGGGMGGMGA3') and 1'R (5'ATCTSTGCTTYGTGGTGGGMGGMGA3'), which were designed from the predicted well-conserved sequences of exon 1 in chicken (Sutrave and Hughes,

Grant sponsor: National Science Council of the Republic of China; Grant number: NSC 87-2311-B002-028-824.

*Correspondence to: Huai-Jen Tsai, Institute of Fisheries Science, National Taiwan University, Taipei, Taiwan, 106.

E-mail: hjtsai@ccms.ntu.edu.tw

Received 11 March 1999; Accepted 18 June 1999 
1989), human (Nomura et al., 1989) Xenopus (Sleeman and Laskey, 1993) c-ski. PCR was carried out for 34 cycles in a $50 \mu \mathrm{l}$ solution containing $500 \mathrm{ng}$ of the oligo $(\mathrm{dT})_{12-18}, 5 \mu \mathrm{l}$ of $10 \times \mathrm{PCR}$ reaction buffer, $1 \mu \mathrm{l} 2.5$ mM dNTP, $3 \mu l 25 \mathrm{mM} \mathrm{MgCl} 2,10 \mu \mathrm{l}$ of each amplifier and 2.5 U Taq polymerase (Promega). Each cycle consisted of $94^{\circ} \mathrm{C}, 1 \mathrm{~min} ; 65^{\circ} \mathrm{C}, 1 \mathrm{~min}$; and $72^{\circ} \mathrm{C}, 2 \mathrm{~min}$. After the RT-PCR product was cloned and sequenced (Sanger et al., 1977), another RT-PCR was performed using primers E-1-5' (5'GAAACGGCTGTGTCTGCCGCAGATTCT3') and P2R (5'AGGYTTCTCYTCYTGYTTCAC3'). Primer E-1-5' was derived from the sequence of the first RT-PCR product using primers $1^{\prime} \mathrm{F}$ and $1^{\prime} \mathrm{R}$. Primer P2R was the most well-conserved sequence of exon 6 of known c-ski.

\section{Rapid Amplification of cDNA End (RACE)}

For the $5^{\prime}$ end, the first strand of cDNA was synthesized with $3 \mu \mathrm{g}$ of total RNA using SuperScript II (BRL) in the presence of $0.1 \mu \mathrm{M}$ of primer $5^{\prime} \mathrm{RA} 1$ (5' GGTCCGCTGCACCTGG3'). The cDNA was homotailed with poly $(\mathrm{dA})$ at the $5^{\prime}$ end using $18 \mathrm{U}$ of terminal transferase Tdt (BM). The resulting tailed cDNA was then used to generate double stranded cDNA by amplification in the presence of $200 \mathrm{nM}$ of each primer RAAP (5'GGCCACGCGTCGACTAGTAC $\left.(\mathrm{T})_{18} 3^{\prime}\right)$ and 5'RA2 (5' GTCGCACACCGAGTTGATCTG3'), $200 \mu \mathrm{M}$ dNTP, and $1 \mathrm{U}$ Taq polymerase (Promega). The $\mathrm{PCR}$ reaction was run over 30 cycles of: $1 \mathrm{~min}$ at $94^{\circ} \mathrm{C}, 1 \mathrm{~min}$ at $64^{\circ} \mathrm{C}$ and $2 \mathrm{~min}$ at $72^{\circ} \mathrm{C}$. The first PCR product were specifically amplified using a nested PCR performed with the RAUAP primer (5'GGCCACGCGTCGACTAGTACT3') and an internal 3' oligonucleotide 1-5'RA3 (5'GGGAGAAATCTCTGAGAACGC3'; nucleotide 859-880) for tski1 or 2-5'RA3 (5'GAAGTCCCGCAGCACCGTGTT3'; nucleotide 709-687) for tski2 under above PCR conditions. The procedure for $3^{\prime}$ RACE was basically as same as that for 5' RACE except that (1) primer RAAP was used to synthesize the first strand of cDNA; (2) the RAUAP primer with either 5'CATGGCTCGGGCTCAGGAGAGG3' for tski1 or 5'CGGTGTCAGCACCAGCCCCT3' for tski2 were used to generate double strand cDNA; (3) the annealing temperature was $70^{\circ} \mathrm{C}$; and (4) the RAUAP primer with either 5'CTTGACACCAAGGAGGCGCG3' for tski1 or 5'GAGGCAGGCGCTGGACAGC3' for tski2 were used in a nested PCR amplification.

\section{Deduced AA Sequence Analysis and Comparison}

The deduced AA sequences of c-Ski from previously investigated species were retrieved from the GenBank (NCBI blast database search) for comparison. Alignment of the sequences was carried out by using the CLUSTAL 4 program (Higgins and Sharp, 1988) of DNASIS (Hitachi software). The weight matrix method (Bishop, 1994) based on the eukaryotic translation initiation context (Kozak, 1978) was used to analyze the initiation codon of TSki1 polypeptide. Chou-Fasman (Chou and Fasman, 1978), Garnier-Osguthorpe-Robson (Garnier et al., 1978) and hydrophobic moment meth- ods (Eisenberg et al., 1984) were used to analyze the secondary structure of Tski polypeptides.

\section{Genomic DNA Extraction and Southern Blot Analysis}

Genomic DNA was extracted from red blood cells of tilapia (Sambrook et al., 1989). For the Southern blot analysis (Sambrook et al., 1989), $10 \mu \mathrm{g}$ of genomic DNA were digested respectively with $B a m \mathrm{HI}$, EcoRI, PstI, and SacI, and hybridized to a $\left[\alpha^{32} \mathrm{P}\right] \mathrm{dCTP}-\mathrm{labeled}$ class-specific probe, which was obtained by digesting plasmid containing RT-PCR product of the tski1 (1290 bp) or tski2 (1452 bp) fragment with NcoI and SepI, respectively. Hybridization was carried out before autoradiography on X-ray film (Kodak BioMax MS-1) for 16 hr at $-70^{\circ} \mathrm{C}$.

\section{Specific Primers for Amplifying tski1 and tski2 From Genomic DNA}

Class-specific primers were used to detect the tski1 and tski2 genes, respectively. For tski1 detection, forward primer 5'GAGTGAGTGGGGAGACAG3' and reverse primer $1-5^{\prime}$ RA3 were used, whereas tski2 detection used forward primer 5'TCCGGGACTGCAACAAACT3' and reverse primer 2-5'RA3. PCR was carried out 2.5 U VioTaq DNA polymerase (Viogene), and consisted of 25 cycles at $94^{\circ} \mathrm{C}$ for $1 \mathrm{~min}$, annealing at $58^{\circ} \mathrm{C}$ for tski 1 or $64^{\circ} \mathrm{C}$ for tski2 for $1 \mathrm{~min}$, and extension at $72^{\circ} \mathrm{C}$ for $1 \mathrm{~min}$. The expected $\mathrm{M}_{\mathrm{r}}$ of the generated PCR products were 688 and $305 \mathrm{bp}$ for tski1 and tski2, respectively.

\section{RNase Protection Assay}

The 1.3 and $1.4 \mathrm{~kb}$ PCR products containing cDNA sequences corresponding to nucleotide 831-2129 of tski1 and nucleotide $659-2120$ of tski2 were inserted into the pGEM-T (Promega) and linearized at the XhoI and $E s p I$ sites to generate two riboprobes of 517 and $340 \mathrm{bp}$, which were respectively designed to protect a $453 \mathrm{bp}$ tski 1 mRNA fragment and a $244 \mathrm{bp}$ tski 2 mRNA fragment. In vitro transcription for tski1 and tski2 were performed with $20 \mathrm{U}$ of T7 and SP6 RNA polymerase (Promega), respectively, in the presence of $50 \mu \mathrm{Ci}$ $\left[\alpha-{ }^{32} \mathrm{P}\right] \mathrm{UTP}$. Total RNA samples $(20 \mu \mathrm{g})$ were extracted from oocytes, stage 15 embryos (freshly hatched embryos), stage 23 larvae (complete yolk-absorption), fry (1-, 2-, and 4-cm body length) and various tissues (brain, eye, gill, heart, intestine, skeletal muscle, stomach, ovary, and testis) using TriZol reagent, and then hybridized overnight at $42^{\circ} \mathrm{C}$ with $10 \mu \mathrm{g}$ of $\left[\alpha^{-32} \mathrm{P}\right] \mathrm{UTP}$ labeled probe with a $5 \times 10^{8} \mathrm{cpm} / \mu \mathrm{g}$ specific activity. RNA-RNA hybrids were digested with RNase A(0.05 U) and T1 (20 U) (Ambion) and analyzed in a 5\% (w/v) polyacrylamide gel. The dried gel was exposed to X-ray film for $2 \mathrm{hr}$ at $-70^{\circ} \mathrm{C}$. For normalization purposes, tilapia $\beta$-actin mRNA hybridized with a $\beta$-actin specific riboprobe served as an internal control. RT-PCR was used to amplify a DNA fragment from total RNA of tilapia larva by using oligo $(\mathrm{dA})_{12-18}$ primer, forward primer 5'TGCGGTATCCATGAGACCAC3' (nucleotide 
2883-2903) and reverse primer 5'GAAGCATTTGCGGTGGACGA3' (nucleotide 3295-3275). The last two primers were corresponding to exons 5 and 6 of the common carp $\beta$-actin genomic DNA (Liu et al., 1990). The 312 bp RT-PCR product was subcloned into the pGEM-T vector and linearized at the DdeI site to generate the riboprobe using T7 RNA polymerase. For quantifying the varying levels of ski expression in the tissues, the image of RNase protection assay data was taken from Kodak DC120 Zoom Digital Camera. Kodak Digital Science 1D Image Analysis Software was use to analyze the mean intensity. The total sum of intensity of two protection fragments resulting from $\beta$-actin probe were counted to normalize.

\section{RESULTS}

\section{Two Classes of Products Produced by RT-PCR}

A 350-bp DNA fragment was amplified after RT-PCR using primers $1^{\prime} \mathrm{F}$ and $1^{\prime} \mathrm{R}$. After subcloning and sequencing, a forward primer (E-1-5') specific for exon 1 of tilapia c-ski was designed to perform another RTPCR with a reverse primer P2R. Two DNA fragments around 1.3 and $1.5 \mathrm{~kb}$ were shown on the gel. The identities of the nucleotide sequence and deduced AA sequence between these two clones were 76.2 and $53.5 \%$, respectively, and we therefore named the $1.3 \mathrm{~kb}$ clone tski1 and the $1.5 \mathrm{~kb}$ clone tski2.

\section{Primary Polynucleotide Sequences and Deduced AA of Tilapia c-Ski DNAs}

Figure 1 depicts the full length polynucleotide sequences and deduced AA sequences of tilapia tski1 and tski2 cDNA. tski1 cDNA contains a single open reading frame (ORF) of 2061 base (nucleotide 541-2601) encoding $687 \mathrm{AA}$ residues. The stop codon TAA is followed by an untranslated region of 572 nucleotides at the $3^{\prime}$ end, in which two potential instability sequences (ATTTA) (Sleeman and Laskey, 1993) and a potential polyadenylation signal are included. tski2 cDNA contains an single ORF of 2057 (nucleotide 370-2511) bases which encode 714 AA residues. At the $3^{\prime}$ end, one potential instability sequence and two potential polyadenylation signals are included.

\section{Comparison of the Deduced AA Sequence of Tilapia TSki1 and TSki2 With c-Ski and SnoN of Some Other Known Species}

In Figure 2, the deduced AA sequences of tilapia TSki1 and TSki2 are compared with those of the Ski proteins of other species. The shared identity between tilapia TSki1 and TSki2 is 57.2\%. Tilapia TSki1 shares $59.7 \%$ identity with chicken c-Ski, $56.9 \%$ with human, and $57.1 \%$ with Xenopus Ski proteins. The corresponding shared identities for tilapia TSki2 are respectively 66.5, 62.7, and 61.3\%. ski-related cDNA, sno, has been reported in humans (Nomura et al., 1989) and chickens (Boyer et al., 1993). However, the AA identity between human SnoN and tilapia TSki1 and TSki2 was as low as 38 and $39 \%$, respectively. The comparison data also reveal that highly conserved domains are located at the termini, exon 1 (AA 1 to 298 of TSki1) and exons 6 and 7 (AA 489 to 655 of TSki1). In contrast, the middle segment of tilapia TSki protein is a highly variable region. Several characteristics of Ski protein found in humans, chickens, and Xenopus are also conserved in tilapia TSki polypeptides. Both TSki1 and TSki2 contained heptad repeats (Nagase et al., 1993), a 25-mer repeat element and an alternating hydrophobic-basic motif at the C-terminus (Sleeman and Laskey, 1993).

\section{Two tski Genes Detected by Southern Blot Analysis and PCR}

Genomic DNA was isolated from tilapia blood, digested with four restriction enzymes, and analyzed using Southern blot hybridization with probes for tski1 and tski2, respectively. The resulting restriction fragment analysis profiles were different (Fig. 3A), and only two common bands were found: an $8 \mathrm{~kb}$ BamHI-band and a $27 \mathrm{~kb}$ EcoRI-band. Genomic DNA was extracted from individual fish and served as a template for PCR amplification using class-specific primers. PCR product around $700 \mathrm{bp}$ was generated using the tski1-specific primers, while the tski2-specific primers generated a product of around $300 \mathrm{bp}$ (Fig. 3B).

\section{Expression of tski1 and tski2 Genes in Various Tissues and During Development}

Tilapia tski genes were expressed in all tissues examined, with the brain and the skeletal muscle containing the most and the least abundant c-ski mRNAs of both classes, respectively (Fig. 4A). In general, tissues such as the heart, intestine, and brain had more tski2 transcript than tski1, and in the testes, where tski2 gene was also expressed dominantly, expression of the tski1 gene was extremely rare. In contrast, in the ovary, tski1 transcript was much more abundant in expression than the tski2 transcript. Figure 4B shows that during development the expression of tski1 appeared to undergo a stepwise decline as embryos developed, with a rapid decline over the hatching stage and relatively little change otherwise. However, the expression of tski2 showed exclusively a high peak at the hatching stage. Consequently, the quantitative ratio of tski1 to tski2 decreased as the tilapia grew to fry of 4-cm body length.

\section{DISCUSSION}

Northern blot analysis of c-ski expression showed that there were two major mRNA species in chickens (Li et al., 1986) and humans (Nomura et al., 1989). This evidence in turn suggests that there might be more than one species of the c-ski gene, resulting either from an alternative splice and/or from two independent genes. Three classes of chicken c-ski cDNA have been found that the multiple cDNAs were derived from alternative splicing (Sutrave and Hughes, 1989). Sleeman and Laskey (1993) also detected two distinct fragments on Southern blot analysis of Xenopus genomic DNA at high stringency, but when the two respective genes were cloned out and sequenced, the 


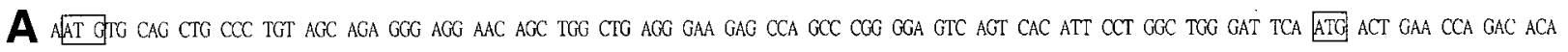
108

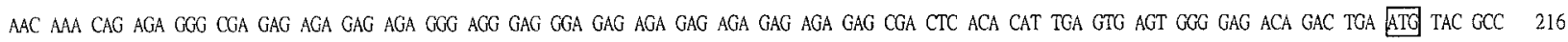
AGA CTG AGC AGA OGA GGC ATA TTT CGG GCG CAC ATT AGG AGT GCG TAA AAC CGC AGG AGC ATA GAa ACA TAG TAC GGC CTG ATT GIA TGT TGG ATA ATA CGA CTT TGG 324 AGA COC TTA CTT GTC GAC TTT AaT TGC GTG TCC TTA CAG GAA CTG CCC TA GATAT TTC AGC AGC TCT CAG TTT TCT GAA TGG ACG CGC TGG GAA GCT TCT CCG GTG ACA 4320 TGG AaG TGC GCG TTA TTG AGC GTG TAG CCG Gat GCT GAG GAC GCC CGA CCC GAT AaG TCG ATT TCA GCT CGC ATT TTG AGT TTT GAA CTC TCT TCT CTA ACC GAC AAC ATG GAG GGC ACG AGC TTC CAG CCC CAT CCT GGa CTT CAC CAa ACT CIG CAG CAG TTT AAT CTG AGT TCC ACO CGC TCC CTC GGT GGa COG GCO GCG TTC TCC GCT CGA MET Glu Gly Thr Ser Ple Gln Pro His Pro Gly Leu Gln Gln Thr Leu Gln Gin Phe Asn Leu Ser Ser Arg Arg Ser Leu Gly Gly Pro Ala Ala Phe Ser Ala Arg

TGG CAC CAG GAC TCA CTC TTC GGG AAA GAC GGC AAA TCC GTC GAG ATG ATG CTC ACC CTG CCG CCT CAG ACA CCT CCA GTG ATG TCC GGT CCA CTT TTC ATC CCG TCC Trp His Gla Asp Ser Leu Phe Gly Lys Asp Gly Lys Ser Val Glu Mel Met Leu Thr Leu Pro Pro Gln Thr Pro Pro Val Met Ser Gly Pro Leu Phe Ile Pro Ser

GAC CGC TCC ACC GAG AGG TGC GAG ACG GTG CTG GAG CGG GAA CCT ATC TCC TGC TTC GTC GTC GGT GGT GAC AAA CGA CTG TGC CTA CCG CAG ATT CTC AAC AGC GTT Asp Arg Ser Thr Glu Arg Cys Glu Thr Val Leu Glu Arg Glu Pro lle Ser Cys Phe Val Val Gly Gly Asp Lys Arg Leu Cys Leu Pro Gln Ile Leu Asn Ser Val

CTC AGA GAT TTC TCC CTC CAG CAG ATC AAC TOG GTG TGC GAC GAT CTC CAC ATC TAT TGC TCC AGC TGC ACA GCG GAC CAG CTA GAG ATC CTC AAA GTG GTG GGT ATC Leu Arg Asp Phe Ser Leu GIn Gin Ile Asn Ser Vai Cys Asp Asp Leu His Ile Tyr Cys Ser Arg Cys Thr Ala Asp Gln Leu Glu Ile Leu Lys Val Val Gly Ile

CTO CCC TTC TCG GCA CCG TCC TGT GGG CTT ATC ACT CAG AOC GAT GCT GAA COG CTT TGC AAC GCG CTC ATC TAT GGC GGT ACT TAC CCT CCT CAT TGC AAC AAG GAG Lcu Pro Phe Ser Ala Pro Ser Cys Gly Leu Ile Thr Gln Thr Asp Ala Glu Arg Leu Cys Asn Ala Leu Ile Tyr Gly Gly Thr Tyr Pro Pro His Cys Asn Lys Glu

TCG GGC TCT CTG GAG TTG GAG CGA ACC GAG AAA AGC TTC AAA GTG TAT CAC CAA TGT TTT GCC COC TGT AAA GGC CTG TTT GTC CCG GAA CTG TAC ACC TCT CCG GGT Ser Gly Ser Leu Glu Leu Glu Arg Thr Glu Lys Ser Phe Lys Val Tyr His Glu Cys Phe Gly Arg Cys Lys Gly Leu Phe Val Pro Glu Leu Tyr Thr Ser Pro Gly

GCC GCC TGC ATC CAG TGC ATG GAC TGC AGA CTC ATG TAC CCA CCT CAC AAG TTT GTT GTC CAC AGT CAC AAG AGA CTA GAG AAC CGG ACA GTC CAC TGG GGC TTT GAT Ala Ala Cys Ile Gln Cys Met Asp Cys Arg Lcu Mel Tyr Pro Pro His Lys Phe Val Val His Ser His Lys Arg Leu Glu Asn Arg Thr Val His Trp Gly Phe Asp

TCT GOC AAC TGG CGG GCT TAT GTG CTC TTA GAC COG GAC TAC ACC GGG AAA GAG GAG AAG AGT CAC CTG GAG AAG CTG CTC AAA GAG TTA AAA GGA AAA TTT GAT CTG Ser Ala Asn Trp Arg Ala Tyr Val Leu Leu Asp Pro Asp Tyr Thr Gly Lys Glu Glu Lys Ser His Leu Glu Lys Leu Leu Lys Glu Leu Lys Gly Lys Phe Asp Leu

ACG GGA AAA CTG TCC AGT AAA TCT TGC AGA TCT CCC AGC COC ATC CGA GCC AAG AGG TCC AAA TTC GAC AAA TTG CAG TCA GCT GAC AAA GAC AGG AAA CCC GAC TGG Thr Gly Lys Leu Ser Ser Lys Ser Cys Arg Ser Pro Ser Pro Ile Arg Ala Lys Arg Ser Lys Phe Asp Lys Leu Gln Ser Ala Asp Lys Asp Arg Lys Pro Asp Trp

CTC CAG TCA CTG TCA AAG TCT GCA CAC AAG GAT CTG AAA CAG GTC CAA CTA AAA CAG AGG CCC TCT GCT TTC CGC CCC TGG TCT CCT AAG CCA GCA GAA AAA GTG AAA Leu Gln Ser Leu Ser Lys Ser Ala His Lys Asp Leu Lys Gln Val Gln Leu Lys Gln Arg Pro Ser Ala Phe Arg Pro Trp Ser Pro Lys Pro Ala Glu Lys Val Lys

CCG GCC CCC AAG AAT GAG GTC GAG AGO TCC TGC TCG AGC AAT CAG GAG ACT CAC AAT TTG GCA TTT GCC CAC CTG GCC CCT GCG GTC CAT GCC AAG GAC AGC AAC ACT Pro Ala Ala Lys Asn Glu Val Glu Arg Ser Cys Ser Arg Asn Gln Glu Thr His Asn Leu Ala Phe Ala His Leu Ala Pro Ala Val His Ala Lys Asp Ser Asn Thr

OCT GAC AGG GGG ACA GCT GCC ATT TCC GTC CAG GAG CTG CAT AAT GOT GAC GCA CAG CCT ACA ACA AAG CCG GCC CAC TCC AGC AAC ACC AAC CGA GCC GAA GAC ATG Pro Asp Arg Gly Thr Ala Ala lle Ser Val Gln Glu Leu His Asn Gly Asp Ala Gln Pro Thr Thr Lys Pro Ala His Ser Ser Asn Thr Asn Arg Ala Glu Asp Met

GAC ACA GAT GGA GAG ATT GAT GTG GAT GAC TGT GAT GAT GGT CCA GTG CAG TCT TCC TCC CTG GCT TCA CCT CCA TCT GCC TGC ACC AGT GTG TCT CAG ACT CTG ACT Asp Thr Asp Gly Glu Ile Asp Val Asp Asp Cys Asp Asp Gly Pro Val Gln Ser Ser Ser Leu Ala Ser Pro Pro Ser Ala Cys Thr Ser Val Ser Gin Thr Leu Thr

CCT CAG AGC ATG GCT CGG GCT CAG GAG AGG CCT TCC TGO CTG CCA GGG ACT GTT TGC CCA GAG ATG GGC ACC ATG AGA CAG ATG CTG TAT GCT GGT CTT GAC ACC AAG Pro Gin Ser Mel Ala Arg Ala Gln Glu Arg Pro Ser Trp Leu Pro Gly Thr Val Cys Pro Glu Met Gly Thr Met Arg Gln Mel Leu Tyr Ala Gly Leu Asp Thr Lys

GAG GCG CGG GAA AAA CTC CTG CAG GAG ATT GTC AGG ATG AGA GTG AAG CAG GAG GAG AAG CTG GCA GCT OCT CTT CAA GCT AAA CGC AGC CTT CAG CAG GAG CTG GAG Glu Ala Arg Glu Lys Leu Leu Gln Glu Ile Va] Arg Met Arg Val Lys Gln Glu Glu Lys Leu Ala Ala Ala Leu Gln Ala Lys Arg Ser Leu Gln Gln Glu Leu Glu

TTT GTG AGA GTC GCT AAG AAA GGG CGT CTT CGC GAG GCC ATC GAG GCC AAG CGC AAC CTG CGA AAG GAG ATC GAA CGC CTT CGC GTG GAC TGG GAG AGG AAG ATG AGG Phe Va] Arg Val Ala Lys Lys Gly Arg Leu Arg Glu Ala Ile Glu Ala Lys Arg Asn Leu Arg Lys Glu Ile Glu Arg Leu Arg Val Asp Trp Glu Arg Lys Met Arg

GAA GCG GAG GAG TCT TGT GGG CGG CTG AAG AGA GAG CTG GAG AGA GAG AGA CAG CTG CGA GTT TGC GAT AAA GGC TGC GAA GCC GAA CGC CTC CGG GTC AAG TAC TCT Glu Ala Glu Glu Ser Cys Gly Arg Leu Lys Arg Glu Leu Glu Arg Glu Arg Gln Leu Arg Val Cys Asp Lys Gly Cys Glu Ala Glu Arg Leu Arg Val Lys Tyr Ser

ACT CAG ATC GAA GAG CTT CAT GTG CAG CTG CAA CAG GCT GAA GCC GAT CGC GAG CAG CTG AGG CGG GAG CTG CAG CAG GAG AGA GAA GCT CGA CAG ACG CTG GAA AGC Thr Gln Ile Glu Glu Leu His Val Gln Leu Gln Gln Ala Glu Ala Asp Arg Glu Gin Leu Arg Arg Glu Leu Gln Gln Glu Arg Glu Ala Arg Gln Thr Leu Glu Ser

GTC GTC AAA GAC CTG CAA ACC CAG CTG GCC CTG CAG GCC GGC AGC ATC CTT CCT GGA GAA TGC AAG GAC ACG AGC ACA GAG GCG CAC AGA CAG ACC GCA CAA CCC ACT Val Val Lys Asp Leu Gln Thr Gln Leu Ala Leu Gln Ala Gly Ser Ile Leu Pro Gly Glu Cys Lys Asp Thr Ser Thr Glu Ala His Arg Gln Thr Ala Gln Pro Thr

AAT GGA TCC TAA AGT TCC ACA TTT AAA ACG AGA CAG AAA GAT TCA GTA AGA GGA GCA TGT TTA AGG GGA AAA AGA AAC AAA GAG TGT TTT TGG TAG GGG GAA AAA AAT Asn Gily Ser ...

CAG TCA TAT ACA ATA TGA TAC AGT ATA CGT CAT TAT TAT TAT GAC ATT ATG AAT TTT ATC AAA TGA ACT GGA AAG CAG CAC GAT GTC CGA GCA TGT AAT AGA GCG TGA AAC TGC GAG AGA CTT TCT TCA GCG ATG GAA AAA CAC ATC TTT CCG GTG ACG TCA GCG CCT GCG GTC AGA CAC CCG ACG CCG TCC AAG GAT TTC ATT TCA GTA TAA GCT ATT CTA AGA TOT GCA AAT ATA GCT ACA TAA CAC TAC TIG AAT ATG AAG GGA AGA AAC ACG TTG CGC CTG GTG CGC AGT TGA TGA AAC TCT TGT TGC CAC ATA TAC TGG TAC ACA CAA GGG TGT CAT TGC AGA CCA CTG GAA ACA AGA GAC ACG AGT TTA CCA CTA TTT ACT TTT AAT ATC TGC TTG CTT TTT ATA ACT GTG CAG AGG ATG TCC TTT

Fig. 1. Polynucleotide sequences of tilapia tski1 (A) and tski2 (B) cDNA and their deduced AA. The nucleotides are numbered beginning with the first nucleotide at the $5^{\prime}$-end, and where it appears, the second smaller number indicates the order of the AA position. The potential initiation codons in the $5^{\prime}$ region are boxed. The potential instability sequences (rapid decay sequences, Sleeman and Laskey, 1993) underscored with wavy lines and the possible polyadenylation signals are underlined. 


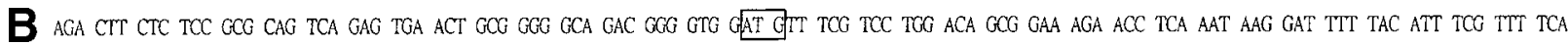
COC CTG ACA AAC GGA ACT TTT CGC CTC GGT GGA GTT TGG AAG CCG TTT TTT CAA ATA TGG ACA GTT GGC GCA CTG TTA CAG GGA GCG ACT GGT CGG TGT TGA CTC ACA

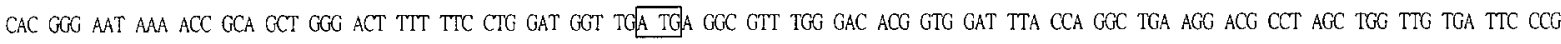

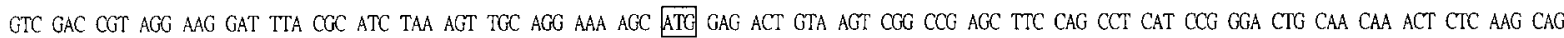
MET Giu Thr Val Ser Ara Pro Ser Phe Gln Pro His Pro Gly Leu Gin Gln Thr Leu Lys Gln

TTT CAC CTC ACC TCT ATG ACC TCG TTG GGT GGA CCC GCC GCT TTC TCT GCT CGC TGG CAA CAT GAG CTG CTC TTT AAG AAG GAC GGG AAG GAG CCC GAG CCG GTT CTG Phe His Leu Ser Ser Mel Ser Ser Leu Gly Cly Pro Ala Ala Phe Ser Ala Arg Trp Gln His Glu Leu Leu Phe Lys Lys Asp Gly Lys Glu Pro Glu Pro Val Leu

CAG CAT CTG CCG COG CCC GTG ATG CCC GGC CCG CTC TTC GTC CCG TCG GAC COC TCC ACG GAG AGG TGC GAG ACC GTC CTG GAG GGG GAG ACC ATC TCC TGC TTT GTG Gin His Leu Pro Pro Pro Val Met Pro Gly Pro Leu Phe Val Pro Ser Asp Arg Ser Thr Glu Arg Cys Glu Thr Val Leu Glu Gly Glu Thr Ile Ser Cys Phe Val

GTC GGC GGG GAG AAA CGS CTC TGT CTG CCG CAG ATT CTC AaC ACG GTC CTG CGG GaC TTC ACC CTG CAG CAG ATC AAC TCC GTG TGC GAC GAG CTG CAC ATC TAC TGC Val Gly Gly Clu Lys Arg Leu Cys Leu Pro Gln Ile Leu Asn Thr Val Leu Arg Asp Phe Thr Leu Gln Gln Ile Asn Ser Val Cys Asp Glu Leu His fle Tyr Cys

TCC COC TGC ACG GCC GAC CAG CTG GAA ATC CTT AAA GTC ATG GGG ATC CTG CCC TTC TCG GCG CCC TCC TCC GGT CTC ATC ACC AAG ACG GAC GCA GaG CGG CTC TGC Ser Arg Cys Thr Ala Asp Glo Leu Glu [le Leu Lys Val Met Gly Ile Leu Pro Phe Ser Ala Pro Ser Cys Cly Leu lle Thr Lys Thr Asp Ala Glu Arg Leu Cys

AaC GCC CTG ATC TAC GGa GGC GCT TAC CCG CCG CGC TGC AAG Aag GAC ATG AAC GGa GGC TCG CTG GAG CTG CAG TTC ACC GAC AGG AGC TTC AAA GTC TAT CAC GAG Asn Ala Leu Ile Tyr Gly Gly Ala Tyr Pro Pro Arg Cys Lys Lys Glu Net Asn Gly Gly Ser Leu Glu Leu Gln Phe Thr Asp Arg Ser Phe Lys Val Tyr His Gln

TGC TTC GGC Aag TGC AAA GGC TTG TTC GTG CCG GaG CTG TAC ACC AGC CCA AAC GCA GCG TGC ATC CAG TGC ATG GAC TCC AGA CTA ATG TAC CCG ACC CAC AAG TTT Cys Phe Gly Lys Cys Lys Gily Leu Phe Val Pro Glu Leu Tyr Thr Ser Pro Asn Ala Ala Cys Ile Gln Cys Met Asp Cys Arg Leu Mel Tyr Pro Thr His Lys Phe

GTG GTG CAC GCC CAC AAG GCG CAG GAG AAC AGO ACT TOC CAC TGG GOC TTC GAC TCG GCC AAC TOG AGG GCO TAC ATC CTC CTG GGC CAG GAT TAC ACG GAG AAA GAG Val Va! His Gly His Lys Ala Gin Glu Asn Arg Thr Cys His Trp Gly Phe Asp Ser Ala Asn Trp Arg Ala Tyr Ile Leu Leu Gly Gln Asp Tyr Thr Glu Lys Glu

GAG AAG GCC CGC CIG GAG CTG TTC CTG GAC GAG ATT AAG GAG AAA TTC GAC TTC GCC AAC AAG TAC AAG AGG AAA GCA TCA TCC AAG GTG TCC GAT CCC ATC CCG GTG Glu Lys Ala Arg Leu Glu Leu Phe Leu Asp Glu Ile Lys Glu Lys Phe Asp Phe Ala Asn Lys Tyr Lys Arg Lys Ala Ser Ser Lys Val Ser Asp Pro Ile Pro Val

aAA AaG TCT AAA CAT GAA GAC CTC TCA TCA CAA ACT CCG CTG GCC GAC AGA GAG AAG CAG CAC GAC TGG CTG CAG TCC CTG TCC ACT CCC AAT AAG GCO CTG AAC TCC Lys Lys Ser Lys His Glu Asp Leu Ser Ser Gin Thr Pro Leu Ala Asp Arg Glu Lys Gln His Asp Trp Leu Gin Ser Leu Ser Thr Pro Asn Lys Gly Leu Asn Cys

ATT CAG TCC ACG CAG AAG CCC TCA GCT TTC AGA CCC TGG TCC CCC CAC ATC TCT GCG GGT GAT AAG GAG CCC TCC AGT GAC COC CTG GCC CTG CTG AGA GAC GGC TTC Ile Gln Ser Arg Gin Lys Pro Ser Ala Phe Arg Pro Trp Ser Pro His Ile Ser Ala Gly Asp Lys Glu Pro Ser Ser Asp Pro Leu Ala Leu Leu Arg Asp Gly Phe

TAC AAT TAC AaG AGC CTA GAG AAG TGG CCC CCA ACG TCG COC TCA CTC CGC TGC CAC TAC GCA AGG TAC GGC CTC TGT CCC CAT CGA TAC CCA CCA CTT CCT ACC CAA Tyr Asn Tyr Lys Ser Leu Glu Lys Trp Pro Pro Thr Ser Pro Ser Leu Arg Cys His Tyr Ala Arg Tyr Gly Leu Cys Pro His Arg Tyr Pro Pro Leu Pro Thr Gln

GTG GAA CCG AAC CTC CAG GTG AGG GCG CAC ACC CCA ACA CCA GAC CTC GCA AGA GGA GAG CTA CCO AGG AGC TCC AGT CCC CAG CAC ATG AAA TCC CTG GGC CTG CTT Val Glu Ala Asn Leu Gln Val Arg Ala His Thr Pro Thr Pro Asp Leu Ala Arg Gly Glu Leu Pro Arg Ser Ser Ser Pro Gln His Met Lys Ser Len Gly Leu Leu

CCT CAG CAG CCA GCA AAC CTC CGC TOC CTC AGS ATG ACA AGS ACT CCO AGO TOG AGA TCG AGS TGG AGA GCC GCG ACG AAG CCO CTT COT CCA TGT CCT CCC TCT GGT Pro Gln Gln Pro Ala Asn Leu Arg Cys Len Are Mel Thr Arg Thr Pro Arg Trp Arg Ser Arg Trp Arg Ala Ala Thr Lys Pro Leu Arg Pro Cys Pro Pro Ser Gly

CGC CAT CTT TTA CCT CAT CCA GCA GCT CAG CCA AAG ACT TCA GCA TCG CCA GOT OTC CAA GCT COC ATC TOC ACT ATC ATC TCA GCC GAA AGC ACG GCA CCC GCA GCC Arg His Leu Leu Pro His Pro Ala Ala Gln Pro Lys Thr Ser Ala Ser Pro Gly Val Gln Gly Pro Ile Cys Thr lle Mel Ser Ala Glu Ser Thr Ala Pro Alá Ala

ACA TOG GTG TCA GCA CCA GCC CCT GTC ACC TCT TCA GAG TCG GCC CTG GAG TCA GAG CTG GAG AGC CTG AGG CAG CCG CTS GAC AGC GGG CTG GAT TCC AAG GAG TCO Thr Ser Val Ser Ala Pro Ala Pro Val Thr Ser Ser Glu Ser Cly Leu Glu Ser Glu Leu Glu Ser Leu Arg Gln Ala Leu Asp Ser Gly Leu Asp Ser Lys Glu Ser

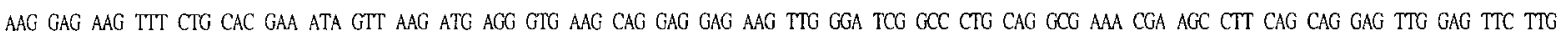
Lys Glu Lys Phe Leu His Glu Ile Val Lys Met Arg Val Lys Gln Glu Glu Lys Leu Gly Ser Ala Leu Gln Ala Lys Arg Ser Leu Gln Gln Glu Leu Glu Phe Leu

COC GTG GCC AAG AAG GAG AAG CTG COG GAG GCG ACC GAG GCA AAG CGC AAC CTC AGG AAG GAA ATT GAG CGT CTG CGA GCT GAG AGT GAG AAG AAG ATC AAG GAA GCC Arg Val Ala Lys Lys Glu Lys Leu Arg Glu Ala Thr Glu Ala Lys Arg Asn Leu Arg Lys Glu lle Glu Arg Leu Arg Ala Glu Ser Glu Lys Lys Met Lys Glu Ala

aAC GAG TCA AGG ATC CGC CTG AaG CGG GAG CTA GaG CAG GCC CGG CAG CTG AGG GTC TGT GAT AAA GCC TGT GAG GCT GGG CGA CTC CGT GCA AAG TAT TCT GCA CAG Asn Glu Ser Arg lle Arg Leu Lys Arg Glu Leu Glu Gln Ala Arg Gln Leu Arg Val Cys Asp Lys Gly Cys Glu Ala Gly Arg Leu Arg Ala Lys Tyr Ser Ala Gln

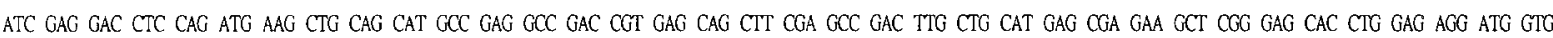
lle Glu Asp Leu Gin Met Lys Leu Gin His Ala Glu Ala Asp Arg Glu Gln Leu Arg Ala Asp Leu Leu His Glu Arg Glu Ala Arg Glu His Leu Glu Arg Met Val

AAG GAG CTA CAG CAG CAA ATC AAG CAC TAA CCT AGA CAG GAC ACC CAA GGA CAT TCC CAT TGG TGA CAA CTA ACC AAG CAG CCA GCC TTA CAG CTG AAC CTT TTC CAT Lys Glu Leu Gin Gln Gin Ile Lys His ...

TTG CCA CCC TGA TCG CCA COC CAC GAG TCC CAC AGG TCG CCC ACA TCA CAC CAC CGT TCC TGT GCT TGC CCC TCA CCC TAC TGA ACA CAA AAC GAC TTC AAA TGO AGA CCC AGA GAA GAA TGG ACA AGC GTT GTC CTT GTG CAT GaA AGG TCT AGT GGT ACC GTA GAA GAA TGA CTG GAA GCA TGC GTT TTC tGa AGC ATT GAC TTT TGC ACC TTC TGA ACT CGT AGA ACT GAG AAC TGG AGC ACA CTG AAC GGC TCT GTA GTG CTG AGT GCG GCT GTG CCT GGA TTT CCT CCA CAA GGA CAC AAG GAG AGC CTG TAG GAC TGT

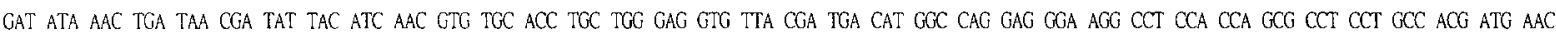

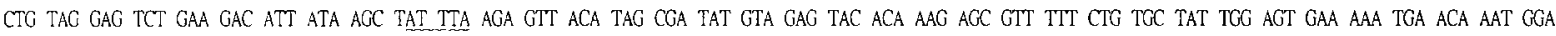
CTT CCC ACT GTG CTG CGA GGS TTT GTC GGT TTG TCA GGA AGC CAT CGC CGC CCA TCG GCC CGA CTC CCC AAA CCA CAA TTG GAT ATT AAA ACA TGG CAA ATG ATT TTA ACA TCA AAG ATT AGG TTT GGC TTT GTG TTC AAC AGG CAC ATT GTC CAT CTC GAA GCC TCA AAA CTG ATC CCT ACC AGT CAC CTG GTG ACG TGT TTT TAA ACA AGC TCT TTT ATT GGA TGC TCT GCA TAC AGC AGC CTC ACA GAC AaG TCG CCA TTT TGT TTG AGC ATT TGA GGT CTG TAC ACA CCC CAG ATG TTT TTG TGC CGT CGC AGT GGG CTG GGT GGT TCG TCT TGT COO TAA CCA CAA ACT GTT CAT TTG AAT TAA ATT AAA GTA TGT ACA TAT AAA TAT

Figure 1. (Continued.) 


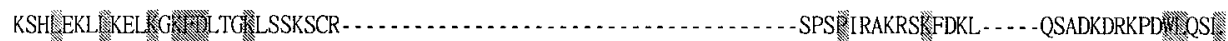

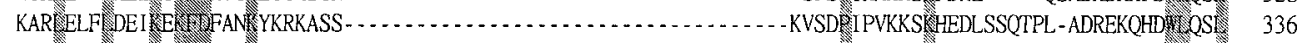

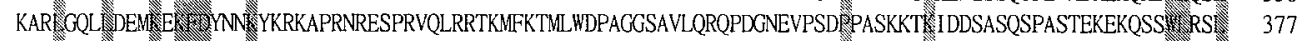

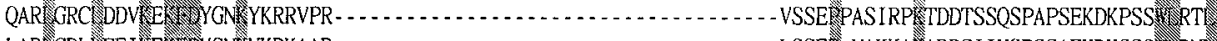

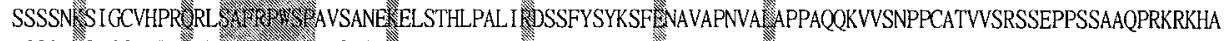

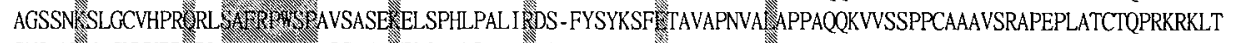

GDAQPTTKPAHSSNTNRAEDMDTDGEIDVD - - - DC - - -DDGP - . -VQSSSLASPSACTSVSQTLTPQSMARAQERP

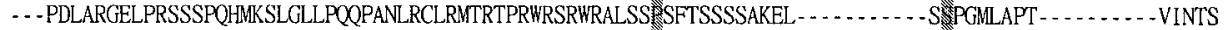

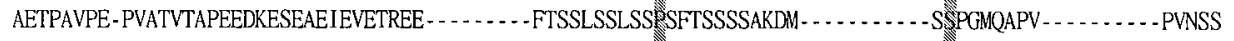

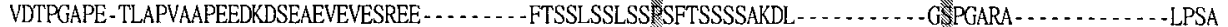
AELPIVPEAPAPPVPI REEEKESETEIEVESREEC ........ . TFTSSLSSATKLLRPCPSGRHLLPHPAAQPKTSA PPGVQPICTIMSAESTAPAATS

$\begin{array}{llr}\text { TSki } 1 & \text { STEAHROTAQPTNGS } & 687\end{array}$

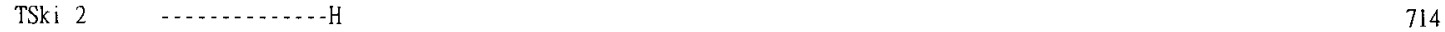

C-Ski NME-......-N $\quad 750$

$\begin{array}{llr}\text { h-Ski } & \text { ELE-.........P } & 728\end{array}$

$\begin{array}{lll}\text { XSki } & \text { DIE-.......-N } & 717\end{array}$

Fig. 2. Comparison of the deduced AA sequences of tilapia TSki proteins (TSki1 and TSki2) with those of chickens (c-Ski), humans (h-Ski) and Xenopus (XSki) Ski proteins. The numbers start at the first AA residue. Identical AA residues are boxed in shadow. Dashed lines

difference between them was only an extra $\mathrm{C}$ residue at position 848. Such a slight difference, however, cannot explain why two RNA bands of about 3.4 and $3.9 \mathrm{~kb}$ were detected when Xski1 was used as a probe in a represent gaps inserted to maximize the identity among all of the sequences compared. The well-conserved AA residues at the tandem repeat domain and at the leucine zipper domain are indicated by hashes (pound signs) and asterisks, respectively.

Northern blot analysis (Sleeman and Laskey, 1993); on the contrary, this latter evidence suggests that another class of Xenopus c-ski remains to be discovered. The present study is the first that clearly demonstrates that 


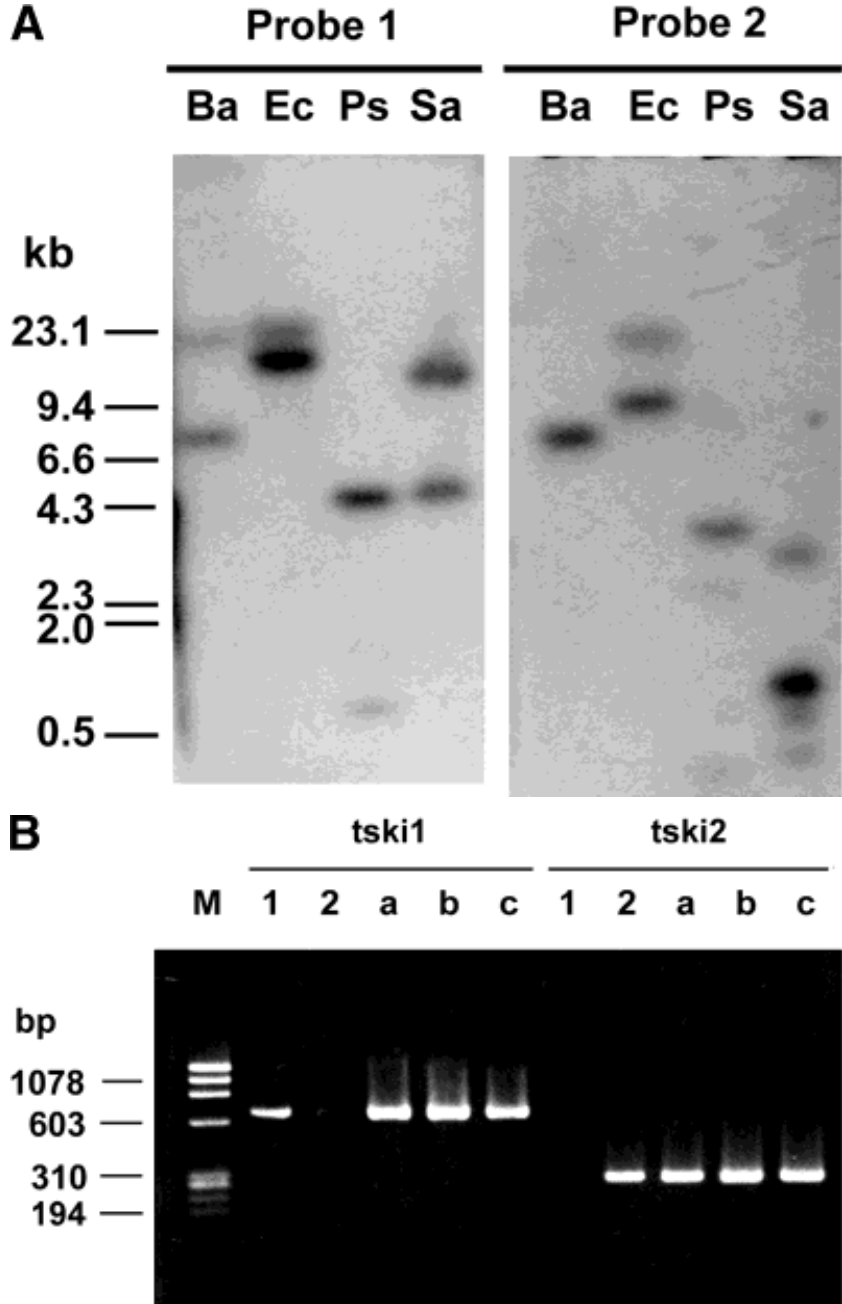

Fig. 3. (A) Southern blot analysis of enzyme-digested genomic DNA isolated from red blood cells of tilapia fry. BamHI (Ba)-, EcoRI (Ec)-, $P s t \mathrm{I}(\mathrm{Ps})-$ and SalI (Sa)-digested genomic DNA were electrophoresed on a gel, transferred to the nylon membrane and hybridized with $\left[\alpha^{-32} \mathrm{P}\right] \mathrm{dCTP}-\mathrm{labeled}$ probe 1 for tski 1 and probe 2 for tski2. Hybridization was carried out in a solution (Sambrook et al., 1989) of 50\% formamide, $5 \times$ Denhardt's solution, $0.5 \%$ SDS, $6 \times$ SSPE and 100 $\mathrm{mg} / \mathrm{ml}$ fragmented calf thymus DNA at $42^{\circ} \mathrm{C}$ for $16 \mathrm{hr}$ with a specific activity of $10^{8} \mathrm{cpm} / \mathrm{\mu g}$. Membranes were washed in $2 \times \mathrm{SSC}$ and $0.5 \%$ (w/v) SDS for $25 \mathrm{~min}$ at room temperature, $2 \times$ SSC and $0.1 \%$ SDS for $20 \mathrm{~min}$ at room temperature, followed by another washing in $2 \times \mathrm{SSC}$ and $0.5 \% \mathrm{SDS}$ for $20 \mathrm{~min}$ at $37^{\circ} \mathrm{C}$. Molecular sizes in kilobase pairs $(\mathrm{kb})$ were given by HindIII-digested lambda. (B) PCR analysis for the presence of tski1 and tski2 in tilapia genome. Genomic DNA was isolated from three individual tilapia fry (lanes a-c) and amplified using specific primers for tski1 and tski2, respectively. Lane $\mathrm{M}$, the molecular marker, HindIII-digested lambda genome; lane 1, plasmid containing tski1 cDNA insert; and lane 2, plasmid containing tski2 cDNA insert.

fish have two distinct c-ski genes. These two genes have their own expression patterns of both in tissues and during development.

Southern blot analysis of tilapia genomic DNA showed that the restriction patterns for the tski1 and tski2 probes were not identical. The tski1 and tski2 probes share common DNA sequences at both ends, corresponding to exon 1 and exons 6 and 7 of c-ski, but vary in the middle segment. The positive bands of the same $M_{r}$
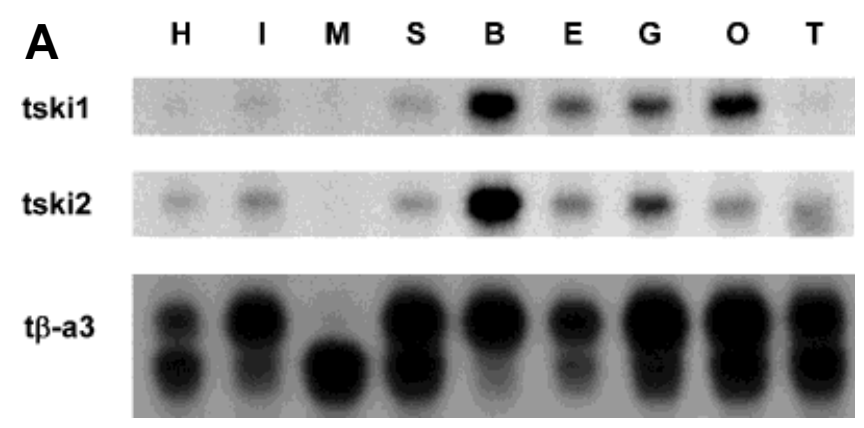

\begin{tabular}{llllllllll}
\hline$\frac{\text { tskil }}{\mathrm{t} \beta-\mathrm{a3}}$ & 0.35 & 0.42 & 0.40 & 0.42 & 0.98 & 0.75 & 0.66 & 0.77 & 0.36 \\
\hline tski2 & 0.46 & 0.50 & 0.37 & 0.46 & 1.13 & 0.71 & 0.69 & 0.58 & 0.63 \\
\hline $\mathrm{t} \beta-\mathrm{a3}$ & & & & & & & & & \\
\hline
\end{tabular}

\section{B \\ Oc Ha La $1 \mathrm{~cm} 2 \mathrm{~cm} 4 \mathrm{~cm}$}

tski1

tski2

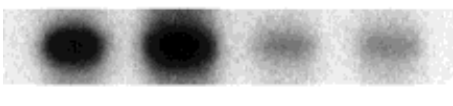

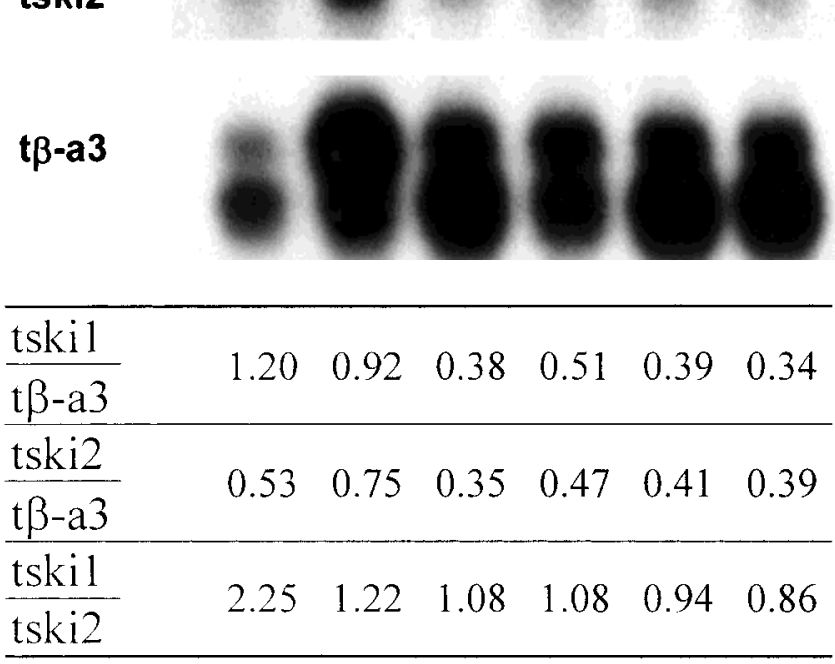

Fig. 4. RNase protection assay for tilapia tski1 and tski2 transcripts in (A) different tissues from adult fish, and (B) different developmental stages. The fragment sizes of tski1 and tski 2 mRNAs protected by tski1- and tski2-specific antisense RNAs were 453 and $244 \mathrm{bp}$, respectively. The protection fragments' size of tilapia $\beta$-actin serving as internal control were 197 and 163 bp. H, heart; I, intestine; M, skeletal muscle; S, stomach; B, brain; E, eye; G, gill; O, ovary; T, testis; Oc, oocytes; Ha, freshly hatched embryos (stage 15); La, larva at stage 23 (yolk absorption); lanes 1, 2, and $4 \mathrm{~cm}$, fry with 1,2 , and $4 \mathrm{~cm}$ body lengths, respectively. Quantified expression levels of ski gene (see Materials and Methods) are presented in tables under the figure as tski 1 over $\beta$-actin (tski1/ $\beta$-actin), tski 2 over $\beta$-actin (tski $2 / \beta$-actin) and tski1 over tski2 (tski1/tski2).

(Fig. 3A) may thus be accounted for by the fact that the two probes may hybridize with a common fragment, although each probe would also hybridize with its own specific bands. The different restriction fragment profiles suggest that there might be two independent c-ski genes. This hypothesis is supported by the PCR results: PCR products of 688 and $305 \mathrm{bp}$ were generated from 
each individual fish when tski1- and tski2-specific primers were respectively employed. All of this evidence strongly suggests that two independent but non-allelic c-ski genes exist in the tilapia genome. Although Northern blot analysis has commonly been used to study the expression and distribution of the c-ski gene in many previous studies, cross-hybridization may prevent this method from being sensitive enough to discriminate between two similar c-ski mRNAs. We therefore used an RNase protection assay with a class-specific RNA probe. The two classes of tilapia tski mRNAs are differentially expressed in some tissues and at certain developmental stages. Thus, like Xenopus Xski1, which is preferentially expressed in the female gonads of Xenopus (Sleeman and Laskey, 1993), tilapia tski1 is expressed at a high level in the ovary, but at a low level in the testes. Surprisingly, however, we found that the tilapia tski2 transcript was extremely rare in the ovary, but absolutely predominant in the testes, an observation that has not previously been reported. In fact, the tilapia tski 1 is maternally inherited, and in this respect it is similar to Xski1 transcripts, which are maternally regulated during early development (Sleeman and Laskey, 1993). Thus as the tilapia embryos develop from oocytes to 4-cm fry and the tski2 transcripts increase, the tsk 1 transcripts decline, presumably because of the degradation of the maternally-inherited stockpile of tski1 mRNA. It should therefore be very worthwhile to investigate what roles tilapia TSki1, an ovary-dominant class, and TSki2, a testis-dominant class, might play in gonad development.

Grimes et al. (1993) reported that in any cell a complete absence of c-ski expression is unlikely or extremely rare. Namciu et al (1995) demonstrated that in mouse embryos at 12.5 days of gestation c-ski was expressed at a high level in the skeletal muscle. On the other hand, some reports stated that c-ski is widely expressed in many different tissues, but they were nevertheless unable to detect either chicken or Xenopus c-ski expression within skeletal muscle (Sleeman and Laskey, 1993; Engert et al., 1995). The present study demonstrated that either tski1 or tski2 gene expression is extremely rare in the skeletal muscle of adult tilapia. Tilapia c-ski transcript can also be detected in the heart, which is consistent with Claycomb and Lanson's (1987) report that c-ski was expressed in rodent cardiac tissue.

Nagase et al. (1993) reported a three tandem repeat of $25 \mathrm{AA}$ at positions 571-645 in human c-Ski. Sleeman and Laskey (1993) stated that Xenopus c-Ski contained a novel sequence of four contiguous 25-mer repeat elements at positions 527-664 which form a pattern of five hydrophobic, one acidic and two basic residues. Meanwhile, Zheng et al. (1997) demonstrated that chicken c-Ski contains five imperfect tandem repeats of 25-mer and formed hydrophobic "bottoms." They proposed that the core consensus of the repeat motif was LXXELEXLR. In this study, however, we found that actually the last three 25-mer repeats (located at AA
535-593 in tilapia TSki1) are quite well-conserved in the c-Ski proteins of humans, chickens, Xenopus, and fish. This fact strongly suggests that LXXELEXXR would more accurately characterize the core consensus sequence of the tandem repeat element at the C-terminus of c-Ski. In this context it is also interesting to note that compared to TSki2, the AA composition of TSki1 is much less similar to the previously known c-Ski proteins. In addition, TSki1 is the only c-Ski protein that lacks the AA 509-529 segment that appears in TSki2. Thus, in TSki1, the first sequence of the five 25-mer tandem repeats is not perfectly conserved.

Based on a computer analysis of the predicted secondary structures of tilapia TSki, we found that the C-terminus (AA 522-659 for TSki1 and AA 539-714 for TSki2) of both classes of TSki encompasses an amphipathic $\alpha$-helical domain, which has a tendency to form a coiled-coil structure. These are characteristics that are conserved in human, chicken, and Xenopus c-Ski sequences, presumably because they are structurally significant and functionally important in Ski proteins. At the C-terminal leucine zipper domain of the Ski protein, there is a periodically arranged AA element, LALELL, which is responsible for dimerization (Nagase et al., 1993) and is also essential for cooperation with the NF1 family of transcription factors (Tarapore et al., 1997). Tilapia TSki1 and TSki2 display a LALELL array at the C-terminus, located in precisely the same position reported for other c-Ski proteins. Because this heptad repeat is well-conserved among Ski proteins, it is highly likely that this sequence may also play an important role in forming the coiled-coil structure of fish TSki polypeptides.

Although tski2 mRNA contains four separate AUGs within the 370-bp segment of the predicted mRNA 5 '-leader, three of these, AUG-1, AUG-2, and AUG-3, located at nucleotide 50,165 , and 253 , would terminate after translation of only 20, 19 , and 40 residues, respectively. Thus, we propose that tilapia TSki2 starts at the AUG located at nucleotide 370. tski1 mRNA, meanwhile, contains eight AUGs within a 540-bp segment of the predicted untranslated region, but again, the reading frame beginning with five of these eight AUGs would give ORF of only a few AA. This leaves AUG-2, AUG-3, and AUG-8, which might plausibly terminate tski 1 cDNA. These AUGs all keep the same ORF and share the same stop codon. Since authentic tilapia TSki1 has not yet been purified, the first AA cannot be conclusively determined. However, we propose that the initiation codon of tski1 mRNA is more likely located at nucleotide 541 (AUG-8), as evidenced from the weight matrix analysis (Bishop, 1994). In this analysis, the score of the AUG-2 sequence (GATTCAATG) was -0.56 . The corresponding score for AUG-3 (AGACTGAATG) was 0.72 , while the AUG-8 sequence (GACAACATG) scored highest with +6.85 . Furthermore, a comparison of the known Ski proteins of humans (Nomura et al., 1989), Xenopus (Sleeman and Laskey, 1993) and chickens (Sutrave and Hughes, 
1989), also suggested that the initiation codon most likely starts at AUG-8.

\section{ACKNOWLEDGMENTS}

The authors thank Dr. Huey-Jen Tsay, Department of Neuroscience, National Yang Ming University, for helping with the RPA experiment; Dr. Ueng-Cheng Yang, Department of Biochemistry, National Yang Ming University; and Mr. Yau-Hung Chen, for assisting in analyzing protein structures.

\section{REFERENCES}

Berk M, Desai SY, Heyman HC, Colmenares C. 1997. Mice lacking the ski proto-oncogene have defects in neurulation, craniofacial patterning, and skeletal muscle development. Genes Dev 11:2029-2039.

Bishop MJ. 1994. Guide to human genome computing. San Diego: Academic Press.

Boyer PL, Colmenares C, Stavnezer E, Hughes SH. 1993. Sequence and biological activity of chicken snoN cDNA clones. Oncogene 8:457-466.

Chou PY, Fasman GD. 1978. Prediction of the secondary structure of proteins from their amino acid sequence. Adv Enzymol 47:45-148.

Claycomb W, Lanson N. 1987. Proto-oncogene expression in proliferating and differentiating cardiac and skeletal muscle. Biochem $\mathrm{J}$ 247:701-706

Colmenares C, Stavnezer E. 1989. The ski oncogene induces muscle differentiation in quail embryo cells. Cell 59:293-303.

Colmenares C, Sutrave P, Hughes SH, Stavnezer E. 1991. Activation of the c-ski oncogene by overexpression. J Virol 65:4929-4935.

Eisenberg D, Sweet RH, Terwilliger TC. 1984. The hydrophobic moment detects periodicity in protein hydrophobicity. Proc Natl Acad Sci USA 81:140-144.

Engert JC, Servaes S, Sutrave P, Hughes SH, Rosenthal N. 1995. Activation of muscle-specific enhancer by the Ski proto-oncogene. Nucleic Acids Res 23:2988-2994.

Garnier J, Osguthorpe DJ, Robson B. 1978. Analysis of the accuracy and implications of simple methods for predicting the secondary structure of globular proteins. J Mol Biol 120:97-120.

Grimes H.L, Ambrose MR, Goodenow MM. 1993. c-ski transcripts with and without exon 2 are expressed in skeletal muscle and throughout chick embryogenesis. Oncogene 8:2863-2868.

Higgins DG, Sharp PM. 1988. CLUSTAL: a package for performing multiple sequence alignment on microcomputer. Gene 73:237-244.

Ichikawa K, Nagase T, Ishii S, Asano A, Mimura N. 1997. Transregulation of myogenin promoter/enhancer activity by c-ski during skeletal-muscle differentiation: the c-terminal of the c-Ski protein is essential for transcriptional regulatory activity in myotubes. Biochem J 328:607-613.

Kozak M. 1978. How do eucaryotic ribosomes select initiation regions in messenger RNA. Cell 15:1109-1123.

Li Y, Turck CM, Teumer JK, Stavnezer E 1986. Unique sequence, ski, in Sloan-Kettering avian retroviruses with properties of a new cell-derived oncogene. J Virol 57:1065-1072.

Liu ZJ, Zhu ZY, Roberg K, Faras A, Guise K, Kapusinski AR, Hackett PB. 1990. Isolation and characterization of beta-actin gene of carp (Cyprinus carpio). DNA Seq 1:125-136.

Ludolph DC, Neff AW, Parker MA, Mescher AL, Smith RC, Malacinski GM. 1995. Cloning and expression of the axolotl proto-oncogene ski. Biochim Biophys Acta 1260:102-104.

Nagase T, Mizuguchi G, Nomura N, Ishizaki R, Ueno Y, Ishii S. 1990. Requirement of protein co-factor for the DNA-binding function of human ski proto-oncogene product. Nucleic Acids Res 18:337-343.

Nagase T, Nomura N, Ishii S. 1993. Complex formation between proteins encoded by the ski gene family. J Biol Chem 268:1371013716.

Namciu S, Lieberman MA, Stavnezer E. 1994. Induction of the c-ski proto-oncogene by phorbol ester correlates with induction of megakaryocyte differentiation. Oncogene 9:1407-1416.

Namciu S, Lyons GE, Micales BK, Heyman HC, Colmenares C, Stavnezer E. 1995. Enhanced expression of mouse c-ski accompanies terminal skeletal muscle differentiation in vivo and in vitro. Dev Dynam 204:291-300.

Nicol R, Stavnezer E. 1998. Transcriptional repression by v-Ski and c-Ski mediated by a specific DNA binding site. J Biol Chem 273:3588-3597.

Nomura N, Sasamoto S, Ishii S, Date T, Matsui M, Ishizaki R. 1989. Isolation of human cDNA clones of ski and the ski-related gene, sno. Nucleic Acid Res 17:5489-5500.

Sambrook J, Fritsch EF, Maniatis T. 1989. In: Nolan C, editor. Molecular cloning. New York: Cold Spring Harbor Press. p 9.1-9.58.

Sanger F, Nicklen S, Coulson AR. 1977. DNA sequencing with chainterminating inhibitors. Proc Natl Acad Sci USA 74:5463-5467.

Sleeman JP, Laskey RA. 1993. Xenopus c-ski contains a novel coiledcoil protein domain, and is maternally expressed during development. Oncogene 8:67-77.

Stavnezer E, Gerhard DS, Binari RC, Batzas I. 1981. Generation of transforming viruses in cultures of chicken fibroblasts infected with avian leukosis virus. J Virol 39:920-934.

Stavnezer E, Barkas AE, Brennan LA, Brodeur D, Li Y. 1986 Transforming Sloan-Kettering viruses generated from the cloned $\mathrm{v}$-ski oncogene by in vitro and in vivo recombinations. J Virol 57:1073-1083.

Sutrave P, Hughes SH. 1989. Isolation and characterization of three distinct cDNAs for the chicken c-ski gene. Mol Cell Biol 9:40464051.

Sutrave P, Kelly AM, Hughes SH. 1990. ski can cause selective growth of skeletal muscle in transgenic mice. Genes Dev 4:1462-1472.

Tarapore P, Richmond C, Zheng G, Cohen SB, Kelder B, Kopchick J, Kruse U, Sippel AE, Colmenares C, Stavnezer E. 1997. DNA binding and transcriptional activation by the Ski oncoprotein mediated by interaction with NF1. Nucleic Acids Res 25:3895-3903.

Zheng G, Blumenthal KM, Ji Y, Shardy DL, Cohen SB, Stavnezer E. 1997. High affinity dimerization by Ski involves parallel pairing of a novel bipartite $\alpha$-helical domain. J Biol Chem 272:31855-31864. 